\title{
Le numérique vecteur d'un renouveau des pratiques de lecture : leurre ou opportunité ?
}

Renewing Reading Practices through Digital Technology: Illusion or Opportunity?

Pierre Barbagelata, Aude Inaudi et Maud Pelissier

\section{(2) OpenEdition}

\section{Journals}

Édition électronique

URL : http://journals.openedition.org/edc/5965

DOI : $10.4000 /$ edc.5965

ISSN : 2101-0366

\section{Éditeur}

Université Lille-3

\section{Édition imprimée}

Date de publication : 1 décembre 2014

Pagination : 17-38

ISBN : 978-2-917562-12-3

ISSN : $1270-6841$

\section{Référence électronique}

Pierre Barbagelata, Aude Inaudi et Maud Pelissier, « Le numérique vecteur d'un renouveau des

pratiques de lecture : leurre ou opportunité ? », Études de communication [En ligne], 43 | 2014, mis en ligne le 01 décembre 2014, consulté le 16 mai 2019. URL : http://journals.openedition.org/edc/5965 ; DOI : 10.4000/edc.5965 


\section{PIERRE BARBAGELATA, AUDE INAUDI ET MAUD PELISSIER LE NUMÉRIQUE VECTEUR D'UN RENOUVEAU DES PRATIQUES DE LECTURE : LEURRE OU OPPORTUNITÉ ?}

\section{Introduction}

Sur le lien entre lecture, livre numérique et adolescents, plusieurs études et recherches récentes font état de constats, qui interrogent la société. La distance prise par les adolescents avec la lecture y est énoncée de manière récurrente (Ertzscheid, 2013). Elle est justifiée par des raisons variées et vraisemblablement complémentaires. En parallèle du «manque de temps », de la «perte d'intérêt» (Evans, 2012), de «l'effet de distanciation face aux injonctions scolaires et/ou familiales » ou encore "d'un phénomène générationnel » (Octobre, 2009), «la montée en puissance de la culture d'écran» et des «pratiques numériques» (Donnat, 2010) est largement convoquée. Ce dernier phénomène, par le type de lecture qu'il entraîne, pseudolecture (Baccino, 2011), fragmentaire (Vandendorpe, 2011) semble aller à l'encontre de la logique d'attention propre au livre imprimé, en particulier chez les plus jeunes.

Dans le même temps, certains types d'œuvres romanesques trouvent leur public, nombreux, fidèle. Et pourtant ils lisent... (Baudelot, 1999) pointait déjà un décalage entre les lectures attendues 
dans le cadre scolaire et les lectures effectives. Une approche comparative des résultats de différentes enquêtes conduites entre 1993 et 2011 (Détrez, 2011), rapporte l'engouement pour la fantasy, les séries, les mangas et l'affaiblissement de la littérature classique ou patrimoniale. Les choix se portent vers des œuvres insérées «dans les circulations entre différents médias»(Détrez, 2011). Déclinées sous forme de produits dérivés, le jeu, la bande dessinée, le film, elles deviennent des objets physiques et numériques autour desquels peuvent se construire des communautés; lesquels entrent en concurrence directe avec le livre en lui-même, invitant à d'autres chemins pour accéder à l'histoire. À l'occasion, elles incitent aussi à un retour au texte.

Aussi, il nous a semblé pertinent de réfléchir à la place, dans l'univers des adolescents, du livre de fiction sur support numérique, ce livre homothétique parfois décrié (Doueihi, 2010) ou une forme enrichie du livre, que nous qualifierons d'euvre l'e-ttéraire. Nous souhaitons en effet considérer ses potentialités (Tréhondart, 2012) afin de ne pas préserver le livre de l'écosystème numérique, afin de ne pas l'écarter définitivement de l'univers culturel des jeunes. Ce faisant nous participons avec cette recherche aux réflexions sur les enjeux communicationnels et sociétaux des médiations numériques en déconstruisant certains schémas établis. Nous cherchons à savoir si l'œuvre l'e-ttéraire en tant que "média informatisé, technique et social, symbolique et culturel » (Jeanneret, 2000) peut devenir le vecteur d'un renouveau des pratiques de lecture pour une population donnée.

Nos propos s'appuient sur les résultats d'une étude empirique à visée exploratoire conduite auprès de lycéens âgés de 15 à 17 ans. Pour une partie d'entre eux, ils ont été mis en situation de lire des cuvres l'e-ttéraires via un dispositif articulant tablettes tactiles, pratiques adolescentes du numérique et médiations humaines. Cette démarche a été conduite dans des classes participant à un prix littéraire et a bénéficié de l'action de médiateurs en charge de l'accompagnement des nouveaux usages numériques en milieu scolaire. Dans ce contexte, nous avons observé, non pas l'acte pédagogique, non pas l'élève en situation d'apprendre une autre manière de lire, mais des pratiques et des comportements signifiants susceptibles d'être judicieusement réinvestis à la fois par les lycéens et par les médiateurs du livre et de la lecture. 
Après un état de l'art faisant émerger les arguments des débats actuels, que pour certains nous questionnons, nous montrerons l'intérêt d'une étude empirique sur cette question. Sans prétendre apporter une réponse définitive, ses résultats viennent relativiser certains constats et proposer des contrepoints optimistes à la fois sur la façon dont les adolescents considèrent la lecture et sur ce que le numérique peut offrir comme opportunités.

\section{La lecture de fiction interrogée par le numérique}

\section{La lecture au cour de politiques publiques du numérique}

Le numérique a la faveur des politiques éducatives et culturelles. Il est, entre autres, perçu comme un moyen de diffusion de la culture et du savoir. Les incitations, les discours, les actions qui le promeuvent sont nombreux et récurrents. Il suffit pour s'en persuader de consulter les portails institutionnels des Ministères de l'Éducation nationale et de la Culture, de lire les plans qui se sont succédés depuis le PAGSI en 1998 (Programme d'action gouvernemental pour la société de l'information). Dès lors, l'École et les bibliothèques notamment, incluent le numérique dans leurs priorités. Parmi les chantiers qui en découlent, l'accès à la lecture sur support numérique commence à occuper une place significative et participe de l'effort constant de l'État consacré au développement de la lecture, depuis la fin des années cinquante (Belisle C., 2004).

Les acteurs de ces politiques publiques sont ainsi invités à proposer des livres, des revues, des journaux, etc. sous forme numérique. Ils s'y investissent tout en étant porteurs d'une conception propre de la littérature et du numérique. Plusieurs aspects de la médiation sont impactés: la mise en espace des lieux, l'accès, l'organisation et le choix des collections et des outils. Le mode de lecture et le contexte importent également. L'École, puisque c'est le domaine support de notre étude, est un lieu où une lecture dite savante (Vandendorpe, 2011) est non seulement apprise et attendue mais aussi comprise et mise en œuvre par les élèves (Belisle, 2004). Ce mode de lecture largement valorisé semble pourtant peu transféré en une pratique sociale courante (Belisle, 2004) non seulement pour la lecture de livres traditionnels mais aussi pour celle en émergence d'euvres l'e-ttéraires. 
Dans ce cadre scolaire, la construction du lien entre les jeunes et la lecture est assumée par une chaîne d'acteurs, allant des enseignants à l'encadrement éducatif et pédagogique, en passant par l'expertise et les actions de partenaires externes à l'établissement scolaire comme le réseau CANOPÉ, les Agences régionales du livre, les éditeurs, les collectivités territoriales. Le rôle de ces médiateurs est fondamental, tant par leur action de mise en contact entre l'œuvre et l'adolescentélève que par les injonctions que leur statut leur permet d'exprimer. Cet ensemble d'acteurs invite mais aussi contraint à la lecture d'œuvres sélectionnées, requises pour la réussite du cursus scolaire ou pour l'enrichissement culturel et l'insertion dans la société.

Au plus près des élèves, tous les enseignants sont concernés depuis que le Socle commun de connaissance, de compétences et de culture 1 a institué dans deux des sept grandes compétences, la «maîtrise de la langue » et la «culture humaniste », « la capacité à lire et comprendre des textes variés » et «l'approche sensible des œuvres». Deux domaines, les lettres et la documentation, se trouvent plus particulièrement au cœur de la réflexion sur l'accès à la lecture via le support numérique. Ainsi l'Inspection Générale de Lettres organise depuis quatre ans un rendez-vous annuel autour des métamorphoses du livre et de la lecture ${ }^{2}$. Et les textes relatifs à la documentation scolaire soulignent le rôle actif que doit jouer le professeur-documentaliste en ce sens ${ }^{3}$. Dans tous ces discours se dessine le rôle favorisant du numérique pour l'insertion professionnelle et sociale. Se dessine aussi l'enjeu essentiel de développer la capacité d'une lecture savante sur ce support (Vandendorpe, 2006).

\section{L'écran contre la lecture?}

Or depuis plusieurs années, d'aucuns évoquent si ce n'est l'antinomie, tout au moins une tension entre l'écran et la lecture savante. Les adolescents «lisent beaucoup plus qu'on ne le pense et l'affirment mais ils lisent autrement et dans un cadre de mutation générale des pratiques de lectures » (Ahr, 2012). Ce que Vandendorpe

\footnotetext{
1 http://www.education.gouv.fr/cid2770/le-socle-commun-de-connaissanceset-de-competences.html

2 http://eduscol.education.fr/pnf-lettres/, site consulté le 11 mai 2014.

3 http://cache.media.eduscol.education.fr/file/actus_2012/77/1/2012_vademecum_culture_int_web_214771.pdf, page consultée le 15 mai 2014.
} 
appelle le mode de «lecture par défaut », largement valorisé par l'École et par la société (Vandendorpe, 2011, 51), autrement dit la lecture de roman, s'efface au profit d'une lecture fragmentaire, ancrée par une activité fréquente et récurrente sur le Web. Une pseudo-lecture (lecture rapide, distractive, discontinue) s'installe rendant toujours plus difficile l'attention et la « lecture profonde et attentive », exigées par la forme livre (Baccino, 2011).

Peut-on pour autant en conclure que la forme roman nécessitant une lecture immersive n'a pas sa place sur le support numérique ?

Alain Giffard a cherché à savoir, si chez les jeunes en particulier «la lecture numérique ${ }^{4}$ peut compenser la diminution de la lecture classique ou [...] si au contraire elle va se développer comme un compartiment d'une culture de l'écran distincte voire opposée à la lecture de l'écrit» (Giffard, 2012, 2013). Plusieurs arguments l'amènent à douter de la première hypothèse et à craindre que si rien ne change la seconde ne devienne une réalité. Tout d'abord, sur le Web, «l'acte de lecture est compliqué et difficile» (visibilité des écrans, typographie, mise en page, absence d'unité) et conduit à une surcharge cognitive. En outre, la lecture sur support numérique se déroule dans un univers d'hyper-attention (en référence à l'expression de la psychologue Katherine Hayles), ou d'attention multitâches, peu favorable à une lecture savante. Enfin, l'univers des outils et des médias numériques, berceau des lectures dites numériques est caractérisé par le fait d'avoir été façonné par des industries dont «l'objectif n'est pas tant de favoriser la lecture que de la détourner vers d'autres choses, la transformer en hits, en points d'accroche de la publicité »(Giffard, 2012). À noter que les adolescents euxmêmes, hors du cadre restreint de la lecture, ont émis un doute sur la possibilité de considérer les technologies numériques, et particulièrement la tablette, comme un support d'apprentissage ${ }^{5}$. Ils croient peu en leur propre capacité à résister au divertissement auquel l'objet les invite.

4 On peut s'interroger sur la pertinence de l'association entre ces deux mots mais ce n'est pas l'objet de l'article. L'expression «lecture numérique » évoque davantage une lecture automatisée par le biais d'un outil comme la vocalisation d'un texte via un ordinateur ou une tablette.

5 Poster : Livre, mobilité et numérique. Une culture entre rupture et continuité, http://emiconf-2013.ens-lyon.fr/posters/p51, page consultée le 15 mai 2014. 
À ces éléments s'ajoutent de virulentes critiques à l'égard de la forme numérique du livre. Selon Milad Doueihi, prendre comme cadre de référence le livre papier est une erreur. Ce dernier, « objet fétiche » et «objet de résistance », ne peut trouver d'équivalent dans le monde numérique (Doueihi, 2010). Il aurait un «design cognitif parfait» (Casati, 2013). À l'inverse, le support tablette favoriserait le zapping et le divertissement (Casati, 2013). Il ne pourrait à ce titre, contribuer à une lecture savante car conçu « pour satisfaire ses besoins rapides et surtout pour en créer continuellement d'autres ». Casati anticipe aussi la disparition du seul support dédié à la lecture de fiction, les liseuses. Non pas en raison de leurs propriétés facilitantes pour la lecture immersive, mais parce qu'elles seraient tombées dans le piège de se rapprocher de l'écosystème des tablettes.

En contrepoint cependant, d'autres pensent que les critiques afférentes à la pseudo-lecture sur écran ne s'appliquent pas automatiquement à la lecture d'un livre numérique. «En opposition aux pratiques erratiques sur internet, le livre numérique se revendique du livre imprimé et de son modèle de lecture, avec de réelles potentialités immersives» (Tréhondart, 2012). Il serait un objet offrant justement la possibilité d'un entre-deux, entre un univers propice à la dispersion (le Web) et un univers, considéré aujourd'hui comme moins attrayant (le livre papier).

À la lecture de ces débats, nous pouvons mieux saisir les enjeux et les tensions liés à la lecture sur supports numériques dans le cadre du protocole proposé. Nous avons choisi de faire reposer notre étude sur des hypothèses bienveillantes où tout n'est pas noir ou blanc, où l'adolescent ne rejette pas la lecture par anticipation, où la version numérique du livre recèle des atouts pour inciter à lire l'e-littérature dans et hors l'École et où les médiateurs peuvent encore jouer un rôle.

\section{Évolution des pratiques de lecture adolescentes : un besoin d'étude empirique}

Sur ces questions, il nous apparait que des apports de nature empirique ne sont pas suffisamment mobilisés. Cette communication s'inscrit dans cette perspective. Dans un contexte spécifique de médiations destinées à promouvoir la lecture chez les adolescents de 15 à 17 ans, notre étude propose une démarche empirique inédite et fait émerger des éléments de réflexion complémentaires. 


\section{Un contexte innovant?}

L'École est coutumière du procédé d'ouvrir une «fenêtre sur le monde » (Moeglin, 2010) en transposant dans les classes des objets communicationnels de la société. Cette démarche se superpose bien souvent avec des discours qui affirment l'innovation comme pierre angulaire de ses évolutions ${ }^{6}$. Il s'agit fréquemment d'éléments pré-existants voire déjà répandus dans la société. C'est plutôt dans l'assemblage et la façon dont leur usage est préconisé que se détermine ou pas le caractère innovant. Il s'agirait d'innovations comportementales (Le Nagard-Assayag, 2011). Pourtant, l'extrapolation dans ce nouveau contexte, scolaire, du cadre de fonctionnement (Flichy, 2003) originel des dispositifs techniques ne garantit en aucune façon la réussite de leur appropriation par les acteurs de l'école. Ces derniers, confrontés à des consignes finalement très scolaires, ne risquent-ils pas de développer un nouveau cadre d'usage convenu dont le prolongement hors du temps scolaire ne pourra pas in fine se concrétiser?

Ainsi dans les lycées supports de l'étude, une bibliothèque numérique est déployée. Elle permet aux élèves et aux enseignants à partir de tout ordinateur, tablette ou smartphone connecté à Internet, de chercher, « emprunter » et lire gratuitement des livres au format epub ou pdf, en ligne ou après téléchargement. Ce dispositif est innovant dans le système scolaire par le fait qu'il permet aux médiateurs (enseignant, professeur-documentaliste) de sélectionner, cataloguer et médiatiser via une interface spécifique des fictions récentes de littérature provenant de différentes plateformes spécialisées ou d'éditeurs. Dans le cadre de notre recherche, il a été décidé, d'une part, d'associer cette bibliothèque à un projet pédagogique de prix littéraire existant depuis plusieurs années. Et, d'autre part, de fournir aux enseignants un lot de tablettes numériques afin qu'ils puissent mener différentes activités de classes. L'objectif est d'inciter les élèves à lire la sélection de romans et de bandes dessinées, à critiquer le récit et à échanger leurs impressions avec leurs pairs jurés littéraires dans et au-delà de la classe.

6 Conseil national de l'innovation pour la réussite éducative : http://www. education.gouv.fr/cid71556/installation-du-conseil-national-de-l-innovation-pour-lareussite-educative.html (site consulté le 31/07/2014). 
Les adultes-médiateurs sont impliqués à différents niveaux de ce projet, de la conception des dispositifs utilisés jusqu'aux activités dans la classe. Toutefois, nous focalisons cette communication sur l'évolution du rapport des adolescents à la lecture sur support numérique dans l'environnement considéré. Nous considérons les actions des médiateurs du point de vue des adolescents et des conséquences qui en découlent pour eux. C'est cette partie du protocole qui est présentée ici.

\section{Méthodologie}

Pour mener à bien cette étude, un protocole d'expérimentation a été mis en place en collaboration avec les équipes enseignantes concernées par le projet de prix littéraire et une équipe du CRDP ${ }^{7}$ responsable des actions de médiation au numérique dans les lycées concernés.

Afin d'évaluer l'influence du dispositif numérique (bibliothèque et tablettes numériques) sur l'évolution de leur appétence pour la lecture d'œuvres de fiction, deux groupes de lycéens ont été distingués, chacun étant placé dans un contexte d'apprentissage à la lecture et d'acculturation numérique différent. Ces deux groupes (appelés gL et $\mathrm{gB}$ ) ont été sélectionnés en raison de leur implication dans leur participation au prix littéraire. Ils appartiennent à deux lycées différents du département du Var qui possèdent des caractéristiques similaires d'accès au livre (sur support papier ou numérique $)^{8}$. Mais seul le premier groupe (gL) a bénéficié d'actions de médiation axées sur la lecture sur tablettes numériques.

L'étude empirique s'est déroulée en trois temps sur une durée de six mois. Dans un premier temps, un questionnaire a été administré aux lycéens des deux groupes visant à dresser un premier panorama de leur conception de la lecture en général, de leurs pratiques sociales du numérique et de leur perception de la lecture sur écran. Il comporte

$7 \quad$ Centre Régional de Documentation Pédagogique, établissement public en charge notamment du développement des usages du numérique à l'École.

8 L'environnement culturel en lien avec le livre présente des similitudes : existence de médiathèques, de librairies (avec un réseau plus dense pour le groupe $\mathrm{gB}$ ), couverture haut débit. Au niveau des lycées proprement dit ils sont de taille équivalente et leurs centres de documentation et d'information disposent d'un fonds documentaire de même nature. 
39 questions réparties en trois volets thématiques : lire, lire sur écran, les pratiques numériques. Il a été administré par un des chercheurs après présentation du projet scientifique à la classe, en précisant bien que ce questionnaire se positionne hors des attendus de la classe et de l'enseignant.

Dans un deuxième temps, une série d'entretiens semi-directifs a été réalisée visant à une meilleure triangulation des données, complétée par l'observation d'une séance spécifique d'utilisation des tablettes (choix d'un titre dans la bibliothèque numérique puis accès et début de lecture).

Enfin, au terme du projet de prix littéraire, les lycéens ont été de nouveau interrogés afin d'évaluer l'impact de cette mise en contexte spécifique de la médiation numérique sur le renouveau des pratiques de lecture. Cela a permis d'éclairer la façon dont le vecteur numérique a été apprécié par les élèves lorsqu'il s'agit de lire une ouvre l'ettéraire. Le questionnaire a été administré dans les mêmes conditions que le premier. Il comporte 32 questions réparties en 3 thématiques : lire et le Prix Littéraire, lire avec des outils numériques, lire aujourd'hui.

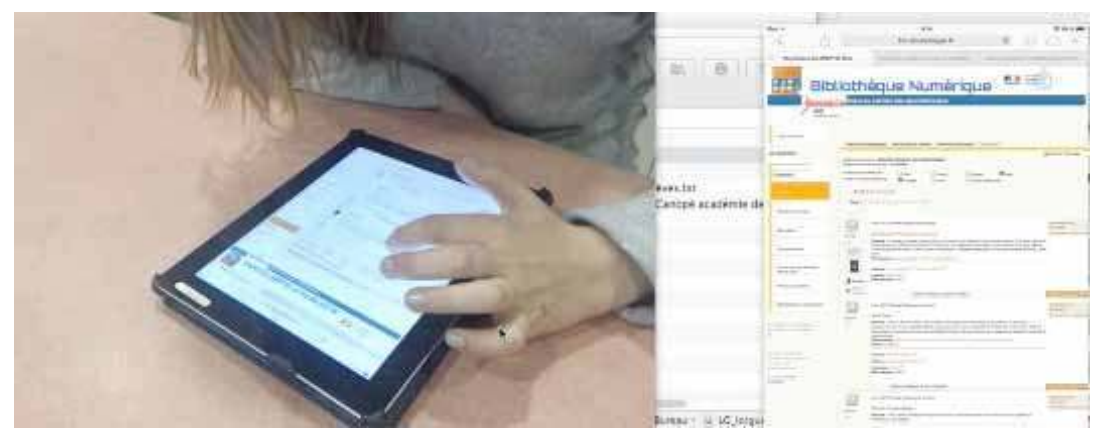

\section{Des résultats en demi-teinte : l'adolescent un lecteur potentiel}

Les adolescents interrogés ont exprimé précisément ce que représente pour eux la lecture de livres. Elle est majoritairement associée à un "petit parallélépipède rectangle », " composé de feuilles de papier » pour l'un, ou encore pour une autre, « racontant une histoire ». À l'heure du numérique, pour une génération dite connectée, leur représentation reste finalement très traditionnelle. Et 
cette lecture, plus appréciée que pratiquée n'est franchement rejetée que pour une faible partie d'entre eux.

\section{Des conditions plutôt favorables}

Les résultats de la première enquête, corroborés par les entretiens semi-directifs, soulignent l'entrelacement intime de conditions à la fois favorables et défavorables à la lecture. Ils confirment l'insertion de la lecture dans un ensemble d'activités possibles impliquant des choix liés au temps disponible et aux priorités (les devoirs, le sport, les sorties...). Comme l'indique un lycéen, qui pourtant peut apprécier la lecture : «c'est une perte de temps, enfin non, ce n'est pas une perte de temps... mais j'ai plein d'autres trucs à faire... le soir, je préfère courir... ». Les pratiques du numérique (musique, réseaux sociaux principalement) se superposent à ces activités mais n'entrent pas, elles, complètement en concurrence.

\section{Une vision positive de la lecture}

Parmi les adolescents interrogés, une majorité exprime un ressenti positif vis-à-vis de la lecture (j'adore : $13 \%$, j'aime bien : 42,3\%). En regard, $25 \%$ seulement revendique un rejet et pour $17,1 \%$, cela dépend des circonstances ${ }^{9}$. Une image positive qui se confirme avec $44 \%$ des mêmes adolescents considérant la lecture d'un livre comme « un moment d'évasion et de plaisir» et comme « un moyen d'apprendre des choses nouvelles» pour près de $27 \%$ (figure 1). Le récit, «s'il me plait, je le finis et je ne me force pas » comme en témoigne une jeune fille qui au demeurant ne s'estime pas particulièrement lectrice.

À l'inverse une minorité, $14 \%$, ressent cette activité comme une contrainte. Ces derniers, à $24 \%$, la lient nécessairement à l'École, ce qui n'est pas le cas pour plus de $84 \%$ de l'ensemble des participants à l'étude. Ce constat semble marquer, pour la plupart des adolescents,

9 Nous avons proposé comme modalités de réponses «j'adore », «j'aime bien », « je déteste », « je ne sais pas », « autre ». Pour ces deux dernières, l'analyse des résultats, les commentaires et les entretiens nous a amené à les regrouper, dans une catégorie « ça dépend ». Ce regroupement n'est pas infirmé par la suite de l'analyse. 
une mise à distance des pratiques de lecture scolaires et des attentes qui y sont liées.

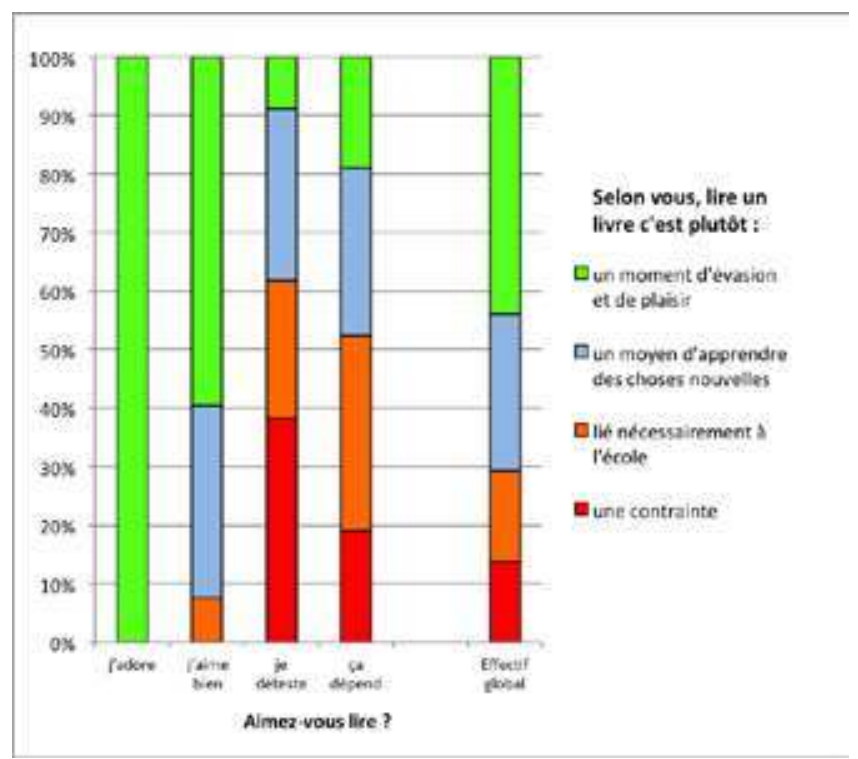

Figure 1 : Goût de lire et ressenti associé

Une relation au numérique intense où s'immisce le plaisir de lire

Les adolescents interrogés sont parfaitement équipés. Tous ont à leur disposition un ordinateur, la plupart, $87 \%$, possèdent un smartphone avec connexion internet et plus de $50 \%$ ont une tablette personnelle et $40 \%$ s'en sont fait prêter une. Ils sont conscients de la prégnance d'internet dans leur quotidien et confirment une lecture par rebond, répondant à une curiosité immédiate.

On ne constate pas chez eux de glissement d'une lecture savante vers une pseudo-lecture. En effet, ceux qui déclarent aimer lire des livres sont aussi ceux qui lisent le plus de pages Web. Symétriquement, ceux qui n'aiment pas lire de livres consacrent moins de temps à la lecture de pages Web (figure 2). 


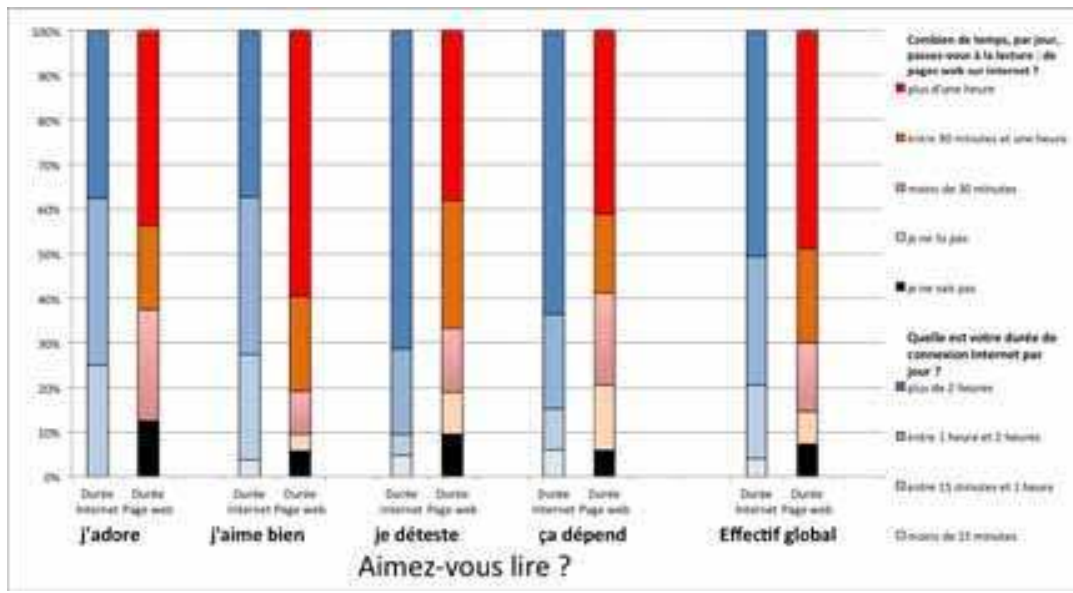

Figure 2: L'importance du Web dans son quotidien et le goût de lire

Les jeunes « réfractaires » à la lecture évoquent la longueur des textes comme un frein, quel que soit le support (livre ou Internet). Un texte long (plus de 10 lignes !) dans un message (courrier, SMS, échanges sur les réseaux) par exemple risque de ne pas être lu en entier. Ils privilégient le morcellement. Un parallèle a d'ailleurs été fait avec les jeux en réseau dont les parties d'un quart d'heure permettent de jouer longtemps. Ces remarques sont intéressantes parce qu'elles proposent une autre approche de la lecture sur écran. Il ne s'agit pas de nier la fragmentation des pratiques de lecture qu'elle entraîne mais de souligner que l'attrait initial pour la lecture joue un rôle dans la capacité d'attention que l'individu est prêt à y investir. En ce sens, les plus gros lecteurs sur papier sont aussi ceux qui pratiquent le plus la lecture sur support numérique.

Deuxième constat, de façon notable, parmi les adolescents interrogés, ceux ayant déjà lu ou commencé à lire une ouvre l'ettéraire sont nombreux (57\%) (figure 3) au regard des pratiques relevées chez les adultes. Cela s'inscrit dans les tendances pointées par les enquêtes IPSOS 2011 et $2014^{10}$ qui relèvent une augmentation

10 Enquêtes 2011 et 2014 : http://www.ipsos.fr/sites/default/files/attachments/ ipsos_livre_hebdo_salon_du_livre.pdf, http://www.ipsos.fr/sites/default/files/attachments/ipsos_cnl_sne_francais_et_lecture2014.pdf. Cette étude ne s'attache pas strictement la tranche d'âge étudiée ici mais pointe pour les plus jeunes (à partir de 15 ans) l'importance de cette dynamique. 
de la lecture de livres numériques en trois ans et un taux plus fort chez les jeunes adultes. On remarque plus précisément, que contrairement au résultat précédent, ceux qui détestent lire sont plus nombreux à lire des livres numériques que ceux qui hésitent ou ceux qui « aiment bien » lire. Les élèves interrogés indiquent que ce support, nouveau tout de même, est essayé en lieu et place du support papier qu'ils ont déjà, véritablement rejeté. Ceux qui adorent lire, le prouvent encore une fois et restent constants dans leur attrait pour le livre, numérique ou papier. Le second questionnaire montre que l'attrait pour la lecture l'e-ttéraire relève plus de la curiosité que d'une pratique de lecture nouvelle.

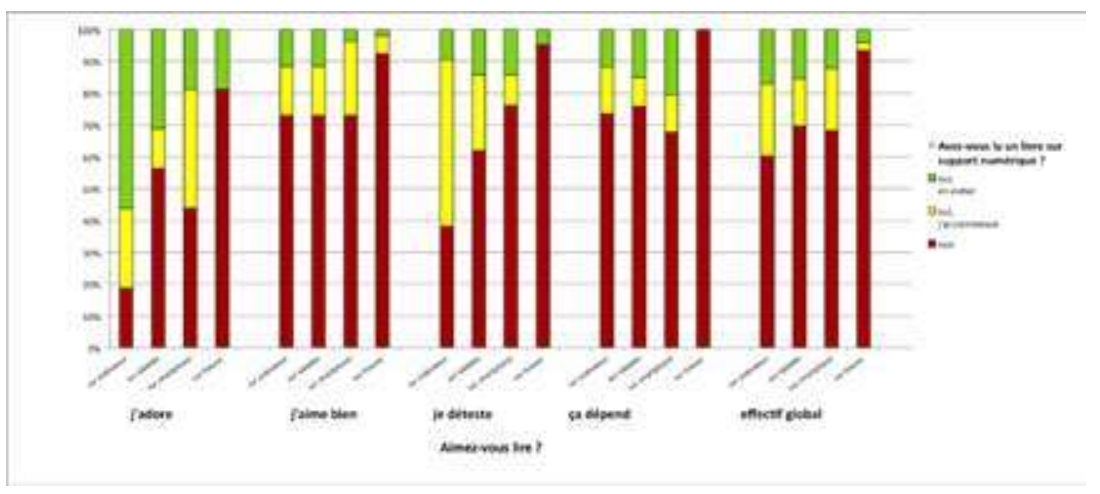

Figure 3 : Avez-vous lu un livre sur support numérique?

\section{Le plaisir d'échanger}

Enfin, cette image globalement positive de la lecture est confirmée par plus de $60 \%$ des répondants qui apprécient échanger sur les livres lus avec d'autres personnes, rappelant en cela que lire est une activité de socialisation. Ce n'est pas qu'une activité solitaire et individuelle mais une activité ouverte au partage et à la communication, comme l'exprime cette lycéenne : "j'ai une copine qui a lu le même livre et on s'échange par textos sur l'histoire ». D'ailleurs leur propension à partager suit relativement leur engouement à lire ; ceux qui adorent le font à $93,8 \%$, ceux qui « aiment bien » à 78,9\%, les indécis à $56 \%$ et ceux qui « détestent » ne sont plus qu'un peu plus de $20 \%$. 


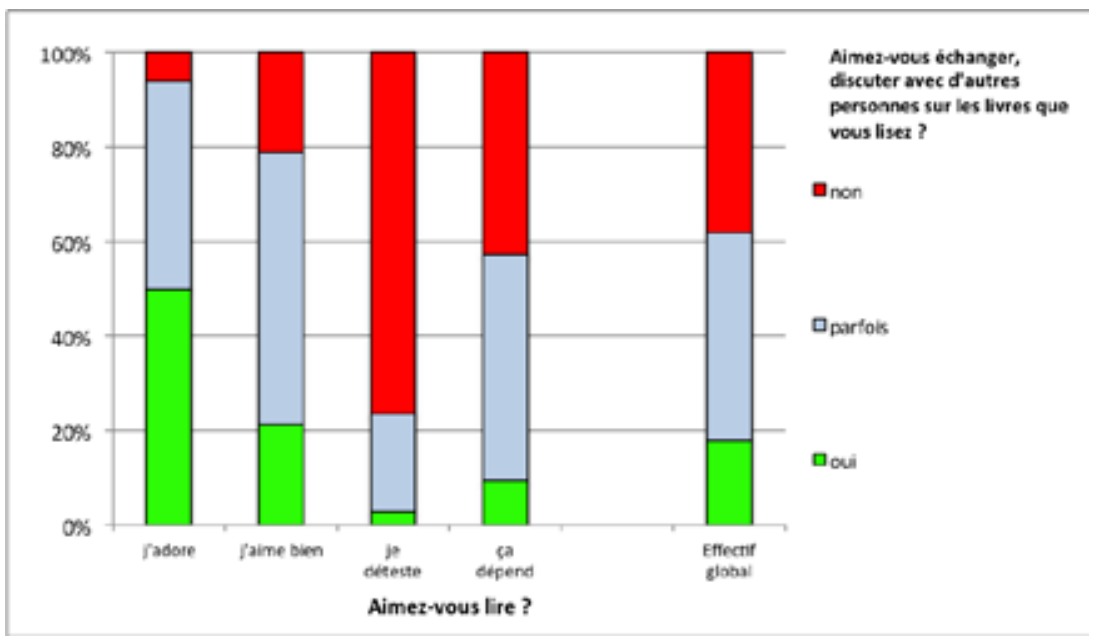

Figure 4 : Lire et échanger

Pourtant le chemin semble encore incertain sur les potentialités sociales du numérique en lien avec la lecture, parfois évoquées ${ }^{11}$. S'ils sont $88 \%$ à utiliser les réseaux sociaux comme un moyen de communication avec leurs amis, près de $70 \%$ disent ne jamais ou presque jamais donner leur avis sur internet (seuls $17 \%$ le font régulièrement). Ils sont d'ailleurs encore $65 \%$ à affirmer ne jamais poser de questions sur internet (forum, blog...). Leur peu d'implication dans le blog du prix littéraire confirme ces doutes. Alors qu'ils ont apprécié l'échange direct autour des œuvres avec leurs enseignants et leurs camarades, près de $90 \%$ d'entre eux n'ont pas contribué au blog du prix. De plus seulement un quart d'entre eux pense qu'utiliser la tablette pour lire faciliterait le partage de leurs impressions avec leurs amis. La dimension communicationnelle du numérique souvent mise en avant, n'apparaît pas, ici, comme un élément de motivation pour l'activité de lecture sur ce support.

11 Cf. un article d'Hubert Guillaud paru sur OWNI en 2011, « le papier contre le numérique », http://owni.fr/2011/03/18/le-papier-contre-le-numerique/ 


\section{Lire selon ses choix}

Certains appellent de leurs vœux, que les « médias informatisés », puissent «aider les enseignants à redonner aux adolescents dont ils ont la charge, le goût de lire et d'écrire, d'écrire pour lire et de lire pour écrire...» (Bizot, 2013). Pourtant, de façon massive, les adolescents revendiquent le choix de lire selon leurs envies. Lire, ou ne pas lire, apparaît comme une affirmation de l'individu autonome qui se construit.

\section{Le choix de lire en se passant de médiateurs professionnels}

D'une part le rôle des médiateurs professionnels (libraires, bibliothécaires, professeurs) est peu ou pas convoqué dans le choix du livre. Même si, paradoxalement, un projet comme le prix littéraire semble une activité pertinente pour les amener à lire des œuvres littéraires. Si la bibliothèque est fréquentée par les gros lecteurs, cela ne signifie pas pour autant que le bibliothécaire est sollicité pour conseiller sur les œuvres à lire. Étonnamment, le CDI de l'établissement n'apparaît pas comme un lieu ressource en matière de lectures de fiction. Et de manière concordante, la bibliothèque numérique de l'établissement est citée par un peu moins de $5 \%$ des élèves ayant lu un livre numérique, malgré le prix littéraire et une présentation en classe de cet accès.

De fait, la lecture effective, quel que soit le support, papier ou numérique, est le résultat d'une envie de l'histoire qu'ils subodorent à partir du paratexte (illustration de la couverture, titre ou encore résumé) et parce que la rencontre opportune avec l'objet «livre » se fait. L'influence du premier cercle relationnel (famille, amis) est significative ( $45 \%$ d'entre eux), allant de $21 \%$ pour ceux qui détestent à $69 \%$ pour ceux qui adorent. Les recommandations issues des forums ou des réseaux sociaux sont «rarement» pour $29 \%$ d'entre eux, et « jamais » pour $52 \%$ prises en compte. Il est notable aussi que la publicité intervient à la marge dans leur choix que le livre soit papier ou numérique. Ces réponses questionnent toutefois si on les met en relation avec le genre de livres lus. À plus de $47 \%$, ils citent les romans fantastiques (héroïc fantasy, fantasy) dont les séries sont fortement présentes sur les étals des librairies physiques et en ligne, qui sont déclinées au cinéma, en jeu vidéo, sites internet... 
Ils semblent totalement faire abstraction du processus marchand dans lequel ils sont insérés. Et dans le même temps en choisissant cette littérature initiatique, peu présente dans le cursus scolaire, ils affirment leur univers propre et leurs choix.

\section{Le choix de lire en évitant les médiations techniques} trop contraignantes

Lors de la première enquête, nous avons noté un a priori positif sur les outils numériques de lecture. Parmi les lycéens interrogés ayant déjà utilisé un ordinateur pour lire un livre, près de $57 \%$ ont trouvé cela agréable ou plutôt agréable. Parmi les $15,6 \%$ qui ont lu un livre en entier sur tablette, $73,6 \%$ ont trouvé cela agréable. Même le smartphone est apprécié par presque la moitié de ceux $(41,6 \%)$ qui ont lu ou commencé un livre sur ce support. À noter le plébiscite pour la liseuse, $100 \%$ de satisfaits parmi les $6,5 \%$ de ceux qui s'y sont essayés.

La concentration et la gêne sont évoquées pour justifier le moindre intérêt pour l'ordinateur et le smartphone. Au final, parmi les supports, seule la tablette semble pouvoir en termes d'attractivité « concurrencer» le livre. Même si la liseuse remplit pleinement sa mission, son attractivité est faible; elle n'est pas perçue pour une majorité comme un objet pouvant s'inscrire dans leur quotidien. 


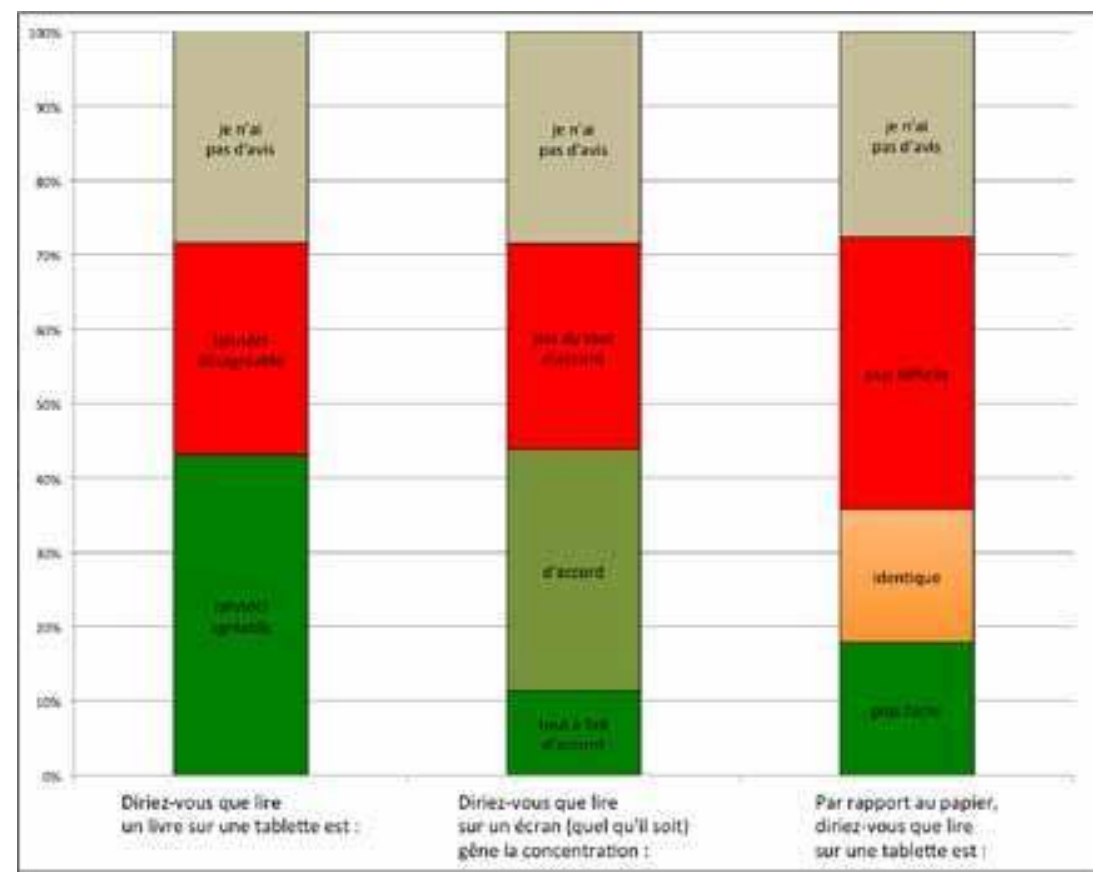

Figure 5 : Perception de la lecture sur écrans

À l'issue de la deuxième enquête et du prêt de tablettes, parmi ceux qui ont commencé un livre numérique sur un support tablette, $60 \%$ déclarent ne pas l'avoir terminé. Ils évoquent comme premiers facteurs d'abandon des caractéristiques propres au support : la fatigue visuelle, le souhait de faire autre chose. Le désintérêt pour l'histoire arrive en troisième position. L'attrait pour cette forme de lecture semble donc avoir diminué en cours de route. Ils étaient $21 \%$ à dire qu'avoir une tablette pourrait leur donner envie de lire. Après avoir expérimenté concrètement cette lecture, ils ne sont plus que $12 \%$. À l'inverse, pour le groupe $\mathrm{gB}$, dans l'établissement n'ayant bénéficié ni du prêt de tablettes, ni d'actions spécifiques de promotion de la lecture sur supports numériques, le mouvement passe de $9 \%$ à $15 \%$. Ces retours laissent entrevoir que l'idée qu'ils se faisaient de la lecture sur tablette, confrontée à la réalité, a perdu de son charme. Ils sont aussi à mettre en regard avec le fait que pour eux terminer un livre même sur papier est loin d'être une évidence (seuls $27 \%$ d'entre eux les terminent systématiquement). 
Nous devons aussi prendre en compte le fait que les tablettes ont été utilisées dans un cadre contraint, celui de l'école, qui n'a pas permis de les mettre dans une situation de pratique quotidienne. La nécessité de lire à des moments imposés par l'enseignant a rendu artificielles des situations de lecture qui s'inscrivaient peu dans le quotidien scolaire et pas assez dans des habitudes sociales. Finalement en rupture avec ce que disent ces adolescents de leurs désirs de lecture. Ils veulent découvrir des histoires à $67,4 \%$, ils le font sur des sites de fiction à $52,4 \%$, par le film, en choisissant ce dernier au détriment du livre si les deux versions existent pour 92,9\% d'entre eux. Ils veulent aussi pouvoir cliquer directement sur le livre au moment où ils le découvrent. S'il faut «attendre », « revenir », « décaler »... alors ils passent à autre chose et oublient. Si l'œuvre l'ettéraire est sur leur trajectoire numérique (les sites qu'ils visitent, le moteur de recherche qu'ils utilisent, les réseaux qu'ils fréquentent...), alors c'est envisageable.

\section{Conclusion}

Au cours de cette étude, nous avons apporté des éléments qui tendent à dépasser un certain nombre d'affirmations dressant entre les adolescents, la lecture et le numérique des frontières rendant impossibles les coexistences. L'adolescent devrait faire un choix : choisir le livre et s'isoler d'un monde numérique en ébullition ou au contraire plonger dans une ère numérique et perdre le livre vecteur culturel d'excellence. En inscrivant notre recherche dans le cadre d'une étude empirique nous avons constaté de fortes similitudes entre leur appréhension du livre papier et celle de la lecture sur support numérique. Tout en considérant la réalité des pseudo-lectures nous soulignons que lire un livre de fiction procède, pour eux, d'une démarche similaire quel que soit le support.

Tout d'abord, cette tranche d'âge apparaît avoir (encore) une appétence pour la lecture, qu'elle ne traduit certes pas toujours en acte. Parmi ceux-ci, certains adorent. Ils lisent par plaisir et intensément, selon leurs choix. Même s'ils préfèrent le support papier, la lecture sur support numérique n'est pas un handicap. Ils cumulent des temps de lecture, livres papier, pages Web et même, livres numériques. Aux médiateurs de savoir leur proposer disponibilité et choix avec les outils de leur quotidien. 
À l'autre bout du spectre se trouvent ceux que l'on pourrait qualifier de réfractaires. La lecture est évitée, quel qu'en soit le support, pages Web ou livre, papier ou numérique et quelle qu'en soit la nature, fictions, documentaires, voire les posts trop longs sur les réseaux sociaux. Pourtant, avec le numérique, ils s'y essayent, presque autant que leurs camarades qui adorent lire. Ce serait le déchiffrage, cette activité qui mobilise totalement leur temps qu'ils évitent. Leur propos n'est pas sans rappeler les épisodes de ces longs romans que les auteurs du XIX ${ }^{\mathrm{e}}$ siècle délivraient journal après journal au fil des semaines et qui ont permis à un grand nombre d'entrer en littérature. Le numérique pourrait être ce supplétif qui dit le texte, le découpe en épisodes, le rend disponible dans ce court temps enfin réservé à cette lecture, peu appréciée honnie, mais tout de même tentée.

Entre ces deux extrêmes, une majorité lit des livres, certes pas intensément, avec ce plaisir d'avoir pu faire au bon moment le bon choix. Il ne faudrait pas grand-chose pour qu'ils lisent plus ou qu'ils lisent moins d'ailleurs : disponibilité, difficulté ou facilité à choisir puis à disposer du livre qui peut leur plaire. Ce comportement, ils l'ont aussi avec le support numérique. Il pourrait apporter cette proximité spatio-temporelle qui rend le récit disponible, ici et maintenant.

Pour les adolescents interrogés, les fonctionnalités, promesses du support numérique sont si évidentes qu'elles ne peuvent être des éléments déclencheurs. Leur habilité opérationnelle, leur fait trouver, malgré tout, la solution pour choisir, accéder et lire «leur » livre s'ils le veulent, vraiment. Et cela, seul l'attrait du récit le détermine. En soi le support numérique n'impacte que faiblement le degré d'appétence pour la lecture de fiction. Il nous apparaît cependant comme une opportunité, voire une nécessité pour que l'œuvre s'inscrive dans leurs trajectoires et qu'ils puissent se l'approprier.

S'ouvre alors une perspective de recherche contribuant à la réflexion sur les dispositifs d'accès aux øuvres e-littéraires intégrant et pleinement intégrés dans les pratiques sociales de lecture et du numérique.

PIERRE BARBAGELATA

Laboratoire I3M -

Université de Toulon

AUDE INAUDI

GRESEC -

Université Grenoble-Alpes

MAUD PELISSIER

Laboratoire I3M -

Université de Toulon 


\section{BIBLIOGRAPHIE}

Ahr S. et al., (2012), Lectures sur écran, lectures sur papier: Discours et représentations des élèves de 15 ans, in : Le français aujourd'hui, $\mathrm{n}^{\circ}$ 178, pp. 65-76.

Baccino T., (2011), Lire sur internet, est-ce toujours lire?, in BBF, $\mathrm{n}^{\circ} 5$, pp. 63-66, http://bbf.enssib.fr/consulter/bbf-2011-05-0063-011, date de la dernière visite : 13 mai 2014.

Baudelot C., (1999), Et pourtant ils lisent..., Paris, Seuil, 245 p.

Belisle C., (2004), La lecture numérique : réalités, enjeux et perspectives, Villeurbanne-Lyon, Presses de l'ENSSIB, 295 p.

Casati R., (2013), Contre le colonialisme numérique. Manifeste pour continuer à lire, Paris, Albin Michel, 208 p.

Détrez C., (2011), Les adolescents et la lecture, quinze ans après, in $\mathrm{BBF}, \mathrm{n}^{\circ} 5$, pp. 32-35, http://bbf.enssib.fr/consulter/bbf-2011-05-0032-005, date de la dernière visite : 13 mai 2014 .

Donnat O., (2010), Les pratiques culturelles à l'ère numérique, in $\mathrm{BBF}, \mathrm{n}^{\circ} 5$, pp. 6-12, http://bbf.enssib.fr/consulter/bbf-2010-05-0006-001, date de la dernière visite : 13 mai 2014.

Doueihi M., (2010), Le livre à l'heure du numérique : objet fétiche, objet de résistance, Marseille, OpenEdition Press, pp. 95-103.

Ertzscheid O., (2013), Numérique. Lecture. Adolescents, Affordance, http:// affordance.typepad.com//mon_weblog/2013/11/numerique-lecture-adolescents.html, date de la dernière visite : 13 décembre 2013.

Evans C., (2011), Quelles attentes pour les bibliothèques en France, in : Evans Christophe (dir.), Lectures et lecteurs à l'heure d'internet: livre, presse et bibliothèques, Éditions du cercle de la librairie, pp. 195-208.

Flichy P., (2003), L'innovation Technique : Récents développements en Sciences sociales vers une nouvelle théorie de l'innovation, Paris, La Découverte, 256 p.

Giffard A., (2012), les industries de lecture : la logique de l'attention, in Dazibao, pp. 39-41, http://www.livre-paca.org/data/dazibaos/pdf/daz32ok.pdf, date de la dernière visite : 13 mai 2014.

Giffard A., (2013), lecture numérique et logique de l'attention, in Dazibao, $\mathrm{n}^{\circ} 36$, pp. 45-48, http://www.livre-paca.org/data/dazibaos/pdf/dazcompletok-reduit.pdf, date de la dernière visite : 13 mai 2014.

Inaudi A., (2008), Ressources numériques à l'École : vers un glissement de la prérogative politique chez les acteurs de l'éducation: Thèse de doctorat de $3^{\text {e }}$ cycle, Marseille: Université Paul Cézanne, Sciences de l'information et de la communication, http://tel.archives-ouvertes.fr/tel-00766441, date de la dernière visite : 14 novembre 2013.

Jeanneret Y., (2000), Y a-t-il (vraiment) des Technologies de l'information?, Villeneuve d'Asq, Presses universitaire du septentrion, $134 \mathrm{p}$.

Le Nagard-Assayage E. et Manceau D., (2011), Le marketing de l'innovation $2^{\mathrm{e}}$ édition - De la création au lancement de nouveaux produits, Paris, Dunod, 353 p.

Moeglin P., (2000), Du mode d'existence des outils pour apprendre, in : Les Enjeux de l'information et de la communication, Varia 2000, 15 p., http://lesenjeux.ugrenoble3.fr/2000/Moeglin/Moeglin.pdf, date de la dernière visite : 13 mai 2014. 
Moeglin P., (2010), Les industries éducatives, Paris, PUF, 127 p.

Octobre S., (2009), Pratiques culturelles chez les jeunes et institutions de transmission : un choc des cultures ?, Culture Prospective, 8 p., http://www2.culture. gouv.fr/culture/deps/2008/pdf/Cprospective09-1.pdf, date de la dernière visite : 13 mai 2014.

Tréhondart N., (2013), Le livre numérique, un objet textuel non identifié, in: Dazibao, ${ }^{\circ} 36$, pp. 42-44, http://www.livre-paca.org/data/dazibaos/pdf/dazcompletokreduit.pdf, date de la dernière visite : 13 mai 2014.

Vandendorpe C., (2006), L'école et la lecture dans le contexte numérique, in: Recherches, $n^{\circ} 44$, pp. 7-15.

Vandendorpe C., (2011), Quelques questions clés que pose la lecture sur écran, in : Belisle C. (dir.), Lire dans un monde numérique, Lyon, Presses de l'ENSSIB, pp. 50-66. 
